Utah State University

DigitalCommons@USU

$1-1-2010$

\title{
Developing High-Performance III-V Superlattice IRFPAs for Defense - Challenges and Solutions
}

Lucy Zheng

Meimei Tidrow

Leslie Aitcheson

Jerry O'Connor

Steven Brown

Follow this and additional works at: https://digitalcommons.usu.edu/sdl_pubs

\section{Recommended Citation}

Zheng, Lucy; Tidrow, Meimei; Aitcheson, Leslie; O'Connor, Jerry; and Brown, Steven, "Developing HighPerformance III-V Superlattice IRFPAs for Defense - Challenges and Solutions" (2010). Space Dynamics Lab Publications. Paper 154.

https://digitalcommons.usu.edu/sdl_pubs/154

This Article is brought to you for free and open access by the Space Dynamics Lab at DigitalCommons@USU. It has been accepted for inclusion in Space Dynamics Lab Publications by an authorized administrator of DigitalCommons@USU. For more information, please contact digitalcommons@usu.edu.

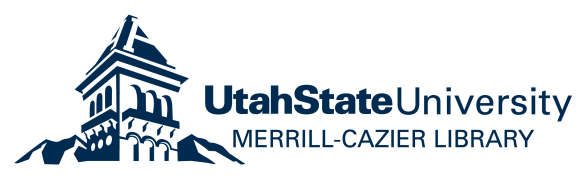




\title{
Developing High-Performance III-V Superlattice IRFPAs for Defense - Challenges and Solutions
}

\author{
Lucy Zheng*a, Meimei Tidrow ${ }^{\mathrm{b}}$, Leslie Aitcheson ${ }^{\mathrm{b}}$, Jerry O'Connor ${ }^{\mathrm{c}}$, Steven Brown ${ }^{\mathrm{d}}$ \\ ${ }^{a}$ Institute for Defense Analyses, 4850 Mark Center Drive, Alexandria, VA USA 22311; \\ ${ }^{\mathrm{b}}$ U.S. Army RDECOM CERDEC NVESD, 10221 Burbeck Road, Ft. Belvoir, VA 22060 \\ ${ }^{\mathrm{c} C o b h a m}$ Analytic Solutions, 7100 Defense Pentagon, Washington DC 20301 \\ ${ }^{\mathrm{d}}$ Space Dynamics Laboratory, 1695 North Research Park Way, North Logan, UT 84341
}

\begin{abstract}
The antimonide superlattice infrared detector technology program was established to explore new infrared detector materials and technology. The ultimate goal is to enhance the infrared sensor system capability and meet challenging requirements for many applications. Certain applications require large-format focal plane arrays (FPAs) for a wide field of view. These FPAs must be able to detect infrared signatures at long wavelengths, at low infrared background radiation, and with minimal spatial cross talk. Other applications require medium-format pixel, co-registered, dual-band capability with minimal spectral cross talk. Under the technology program, three leading research groups have focused on device architecture design, high-quality material growth and characterization, detector and detector array processing, hybridization, testing, and modeling. Tremendous progress has been made in the past few years. This is reflected in orders-of-magnitude reduction in detector dark-current density and substantial increase in quantum efficiency, as well as the demonstration of good-quality long-wavelength infrared FPAs.

Many technical challenges must be overcome to realize the theoretical promise of superlattice infrared materials. These include further reduction in dark current density, growth of optically thick materials for high quantum efficiency, and elimination of FPA processing-related performance degradation. In addition, challenges in long-term research and development cost, superlattice material availability, FPA chip assembly availability, and industry sustainability are also to be met. A new program was established in 2009 with a scope that is different from the existing technology program. Called Fabrication of Superlattice Infrared FPA (FastFPA), this 4-year program sets its goal to establish U.S. industry capability of producing high-quality superlattice wafers and fabricating advanced FPAs. It uses horizontal integration strategy by leveraging existing III-V industry resources and taking advantage of years of valuable experiences amassed by the $\mathrm{HgCdTe}$ FPA industry. By end of the program span, three sets of FPAs will be demonstrated - a small-format long-wave FPA, a large-format long-wave FPA, and a medium-format dual-band FPA at long-wave and mid-wave infrared.
\end{abstract}

Keywords: Infrared focal plane arrays (IRFPAs), infrared detectors, antimonide superlattice, Rule 07, III-V semiconductor foundry, InAs/GaSb, InAs/InGaSb, FastFPA

\section{INTRODUCTION}

Driven by the need for high-performance infrared focal plane arrays (FPAs), the US government has invested in various new infrared detector materials and FPA technologies in the past decade, including HgCdTe, quantum well infrared photodetectors, dilute nitride, and antimonide superlattice. The most important criterion for these technologydevelopment programs is that the FPAs must meet the diverse defense applications. Some of the common FPA features derived from these application requirements are large format to support wide field of view at long wavelengths with near background limited performance (BLIP) and with minimal spatial cross talk, medium format dual-band pixel coregistered FPAs with BLIP performance and minimal spectral cross talk, radiation-hardened detector materials as well as readout integrated circuits (ROIC) and FPA chip-assembly packages. In addition, long-term research and development cost, product availability, and industry sustainability are also important factors in choosing a winning technology.

Investment in antimonide superlattice infrared materials has started since 2003. In 2005, a focused research program was established to develop antimonide superlattice infrared detector technology. The justification for this choice has been

Infrared Technology and Applications XXXVI, edited by Bjørn F. Andresen, Gabor F. Fulop, Paul R. Norton, Proc. of SPIE Vol. 7660, 76601E · (c) 2010 SPIE · CCC code: 0277-786X/10/\$18 · doi: 10.1117/12.852239 
discussed elsewhere and will not be repeated here. ${ }^{1}$ Three major research teams were funded for this work-Naval Research Laboratory and Teledyne Imaging Sensors (NRL/TIS), Jet Propulsion Laboratory and Raytheon Vision Systems (JPL/RVS), and Northwestern University and MPT Technologies (NWU/MPT). The technology program is augmented by including research efforts at small businesses and universities funded through Small Business Innovation Research (SBIR), Small Business Technology Transfer (STTR), and other programs. Most data presented in this paper are from the three major teams.

With the rapid progress made under the technology program, it is recognized that further innovation and development necessitate the participation of U.S. industry members. The research institutions are not equipped to produce large numbers of wafers in a short period of time. However, to develop new processing technologies and protocols, they have to consume many wafers and carry out extensive experiments and tests. For the HgCdTe FPA industry, there are no commercial wafer suppliers because there are no diverse commercial applications of HgCdTe FPA to be profitable. The wafers are grown within each FPA fabrication facility (or its exclusive partner). In other words, the HgCdTe FPA industry is vertically integrated. There are advantages for such an arrangement, such as close interaction between material growers, designers, and FPA processing and testing engineers, but one important disadvantage is the high cost. Our solution is to embrace horizontal integration, which is especially effective for avoiding the initial heavy investment in capital equipment and subsequent upgrading and maintenance, as well as the cost of highly skilled engineers and technicians. The high-cost hurdle is especially difficult to overcome in today's environment, particularly in a situation of developing an alternative technology to compete with a fairly mature and widely deployed technology. Fortunately, there are many commercial III-V semiconductor wafer foundries, mainly supported by the telecommunications industry. These profitable vendors can use their existing wafer production equipment for antimonide superlattice material growth with minimal initial investment. The use of multiple-wafer MBE machines could produce, with good uniformity and repeatability, many more wafers to satisfy the demand, which is impossible when using research-purpose MBE machines.

It is also recognized that the experienced $\mathrm{HgCdTe}$ FPA fabrication industry can offer valuable technology knowhow and infrastructure when developing antimonide superlattice FPAs. The incumbent and new FPA vendors can contribute significantly in device architecture design, etching, hybridization, substrate thinning and removing, FPA characterization and testing, and chip-assembly packaging. If we could adopt a horizontal integration model (e.g., let the III-V industry supply wafers to these FPA fabrication houses), we could reduce the initial research and development cost. This may give the antimonide superlattice development program a better chance to succeed in today's environment.

In spring 2009, the Fabrication of Superlattice Infrared FPA (FastFPA) program was established. This 4-year program sets its goal to establish a U.S. industry capability of producing high-quality superlattice wafers and fabricating advanced FPAs. By end of the program span, three sets of FPAs will be demonstrated - a small-format long-wave FPA, a largeformat long-wave FPA, and a medium-format dual-band FPA at long-wave and mid-wave infrared. Detailed discussion will be given in Section 4.

\section{PROGRESS IN DETECTOR AND FPA PERFORMANCE}

\subsection{Dark current density and Rule 07}

At the detector level, performance is measured by two important parameters - dark current density and quantum efficiency (QE). The absorption coefficient for antimonide superlattice at long wavelengths can reach $2000 \mathrm{~cm}^{-1}$ near cutoff wavelengths. ${ }^{2}$ High quantum efficiency FPA can be achieved with backside illumination if the absorber thickness is larger than $6 \mu \mathrm{m}$, provided the absorbing substrate is thinned or removed and antireflection coating is applied, and the minority carrier diffusion length is comparable to absorber thickness for efficient collection of photocurrent. A FPA with a quantum efficiency of $72 \%$ was reported, with an absorber thickness of $6.5 \mu \mathrm{m} .{ }^{3}$ It was also reported that dark-current density is insensitive to device thickness when operated near tunneling regime. ${ }^{4}$ The increase in dark-current density with absorber thickness is an issue in general, however, and should be addressed.

Rule 07 was first presented by William Tennant at the 2007 U.S. Workshop on the Physics and Chemistry of II-VI Materials. The revised version was presented at the workshop of the same title held in 2009. The detailed description of Rule 07 and data used for deriving it can be found in the two papers and references listed in those papers. ${ }^{5}$ Rule 07 is a simple empirical relationship that describes the dark current behavior with temperature and wavelengths. It is derived from 67 pieces of epitaxially grown materials. All diodes and arrays were grown with MBE and used double-layer planar heterostucture (DLPH) architecture. These layers' diodes were among the best ever made, using a well-controlled 
$\mathrm{HgCdTe}$ photodiode processes. Rule 07 is a convenient rule of thumb for estimating the dark current density for state-ofthe-art $\mathrm{HgCdTe}$ photodiodes, with an accuracy of $0.4-2.5 \times$ the calculated value (see equation 1) over 13 orders of magnitude. It is valid for cutoff wavelength and temperature product larger than $400 \mu \mathrm{m}-\mathrm{K}$. Some data points for very long wavelengths were taken from devices operating at $60 \mathrm{~K}$.

Since all data in this paper were taken at long-wave infrared spectral band, the following equation and parameters are used to calculate the dark current density under Rule 07 :

$$
J_{d}=J_{0} e^{C(1.24 q / k \lambda T)}
$$

where

$$
\begin{aligned}
& J_{0}=8367.00001853855 \mathrm{~A} / \mathrm{cm}^{2}, \\
& C=-1.16239134096245, \\
& q \text { is electron charge, } \\
& k \text { is the Boltzmann's constant, and } \\
& T \text { is the operating temperature. }
\end{aligned}
$$

\subsection{The performance of single-element detectors}

We measure our program's progress against Rule 07 because this is a simple way to compare detector dark current performance at different temperatures and cutoff wavelengths. Figure 1 shows superlattice detector performance measured by dark current density as a multiple of Rule 07 versus time in years. All data plotted here are from singlecolor detectors with GaSb substrate and published in the open literature. The time axis refers to either the paper submission date or the presentation date if it was published in conference proceedings. Note that we used the following criteria when selecting data:

- Only those with complete sets of performance parameters are used for this plot, including at least operating temperature, cutoff wavelength, bias voltage, and dark current density.

- Many early-year papers reported the zero-bias dynamic resistance-area product $R_{0} A$ value instead of darkcurrent density. For homojunction devices, dark-current density $J_{d}$ is converted from $R_{0} A$ values using the equation $J_{d}=k \cdot T /\left(q \cdot R_{0} A\right)$.

- Cutoff wavelength is defined as the wavelength where QE falls off to $50 \%$ of its plateau. In a few cases where quantum efficiency data are not available, the cutoff wavelength is extracted from responsivity data. ${ }^{6-27}$

From Figure 1, we can see tremendous progress made in the past few years on single element detectors or mini-arrays from our major research institutions (i.e. Northwestern University, Naval Research Laboratory, Jet Propulsion Laboratory, and Teledyne Imaging Scientific). The black arrow is an eye aid to see the trend (i.e., about three orders of magnitude reduction in dark current density within the past 10 years). The best device is within three times the value of Rule 07 . We should point out that only a few detectors have an absorber thickness exceeding $6 \mu \mathrm{m}$, as indicated by the large circles. Most of the materials cited here have an absorber thickness of 2-4 $\mu \mathrm{m}$, which limits its reported quantum efficiency number to less than $40 \%$. However, the general trend revealed here about dark current density reduction is valid.

One of the most important contributors to the dark current reduction is the advanced device architecture designs that take great advantage of bandgap engineering available to superlattice materials. During the early years, simple P-N and P-I-N homojunction designs were used by most groups. Innovative designs such as $\mathrm{W}$-structure from NRL, M-structure and PM-P structure from NWU, and complementary barrier infrared detector (CBIRD) structure from JPL enabled orders-ofmagnitude reduction in dark current density caused by G-R, tunneling, and surface leakage. More sophisticated variations of these structures have been realized to reduce the dark current without sacrificing quantum efficiency.

Another important contributor is superlattice material quality improvement, which is quantitatively gauged by several parameters. From high-resolution X-ray diffraction (HRSXD) rocking curves, the mismatch between 0th order superlattice and substrate layers can be extracted from diffraction peak positions, and the combined effect of strain and defect may be reflected in the full width at half maximum (FWHM) of the superlattice diffraction peaks. Device structural information such as superlattice period can also be derived and compared with target numbers. Typical X-ray- 
derived numbers show that the mismatch between superlattice and substrate layers is about 100-700 pm, the FWHM of the superlattice 0th order peak is about 20-30 arcsec, the difference in the designed superlattice period and the measured value is about $3 \%$. Images taken with Normaski microscope, scanning electron microscope, and atomic force microscope (AFM) provide information about surface morphology information, which is correlated with electrical performance. ${ }^{49}$ Typical values achieved by the major research teams are $1-3 \AA$ in AFM RMS for a $10 \times 10 \mu \mathrm{m}$ sample.

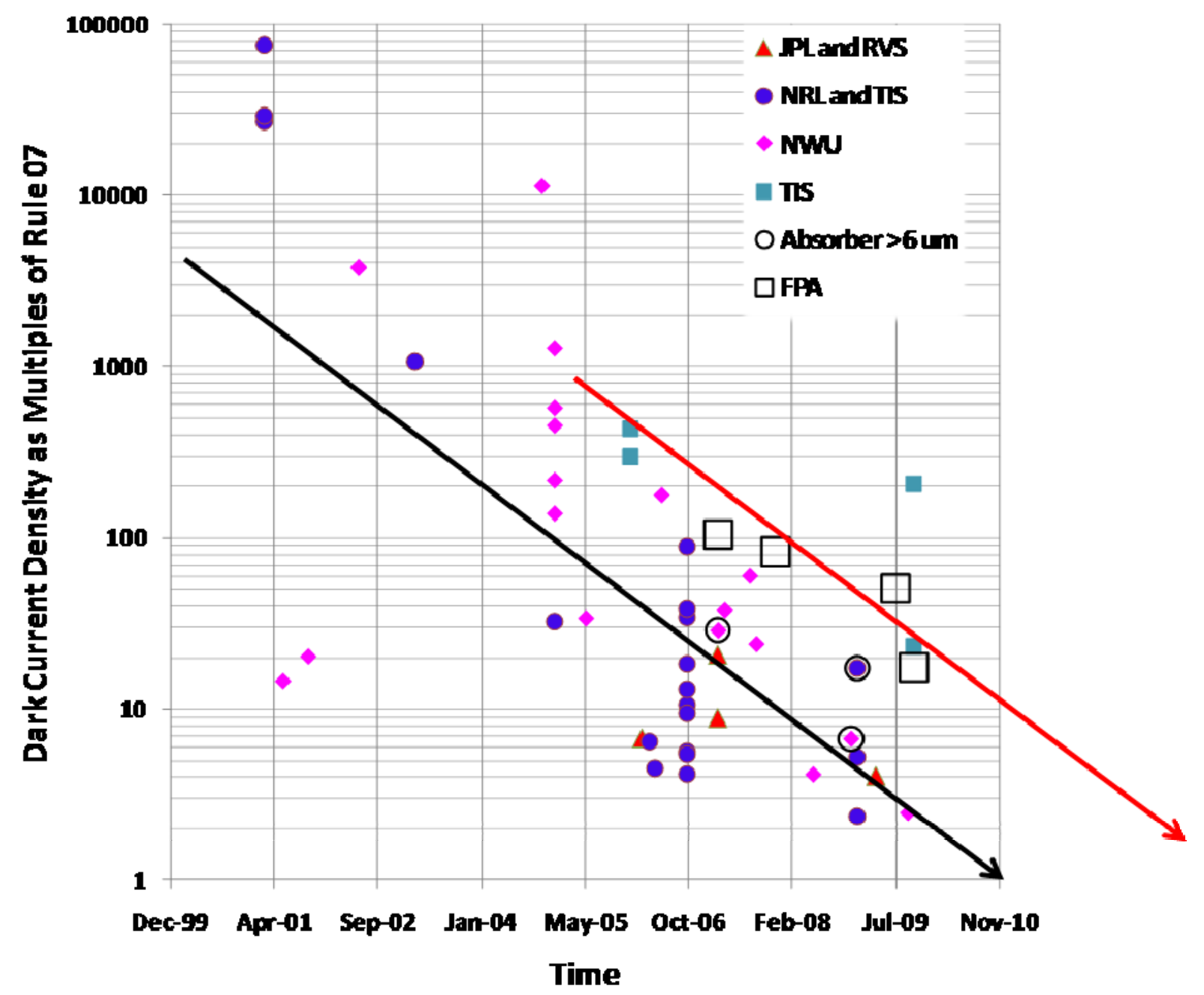

Figure 1: Detector dark current density as a multiple of Rule 07 vs. time. The long black line is drawn as an eye aid to show the trend of dark current reduction along time for single-element detector, and the shorter red line helps to show the trend for FPAs.

\subsection{The performance of focal plane arrays}

Few fully characterized data about superlattice FPA performance at long wavelengths appear in the open literature. Only four data points are plotted in Figure 1, as denoted by the large unfilled squares. ${ }^{28-33}$ They represent the average dark current density of these FPAs. The lowest dark current density is within a factor of 20 of Rule 07 . The red arrow helps show the trend of reduction over time. We can see that at the FPA level the dark current density is about one order of magnitude higher than that of single-element detectors. Note that the two FPAs published in 2009 have thicker absorber layers, $6.5 \mu \mathrm{m}$ and $8 \mu \mathrm{m}$. The reported quantum efficiencies for these two FPAs are $72 \%$ and $33 \%$, respectively. The lower quantum efficiency is partly due to light absorption by the thick substrate. 
Also note that when reporting single-valued quantum efficiency, there is no standard definition to follow. Some people report the peak value, others report the value at the wavelength of a narrow-band spectral filter used for radiometric calibration. To equitably compare results from different groups, we recommend using the following definition. As illustrated in Figure 2, the single-valued quantum efficiency is defined as the wavelength equal to $80 \%$ of the cutoff wavelength. At the cutoff wavelength, the quantum efficiency falls to $50 \%$ from the plateau. The quantum efficiency refers to the number of photoelectrons per incident photon. It should be distinguished from responsivity, which is in units of amperes per watt.

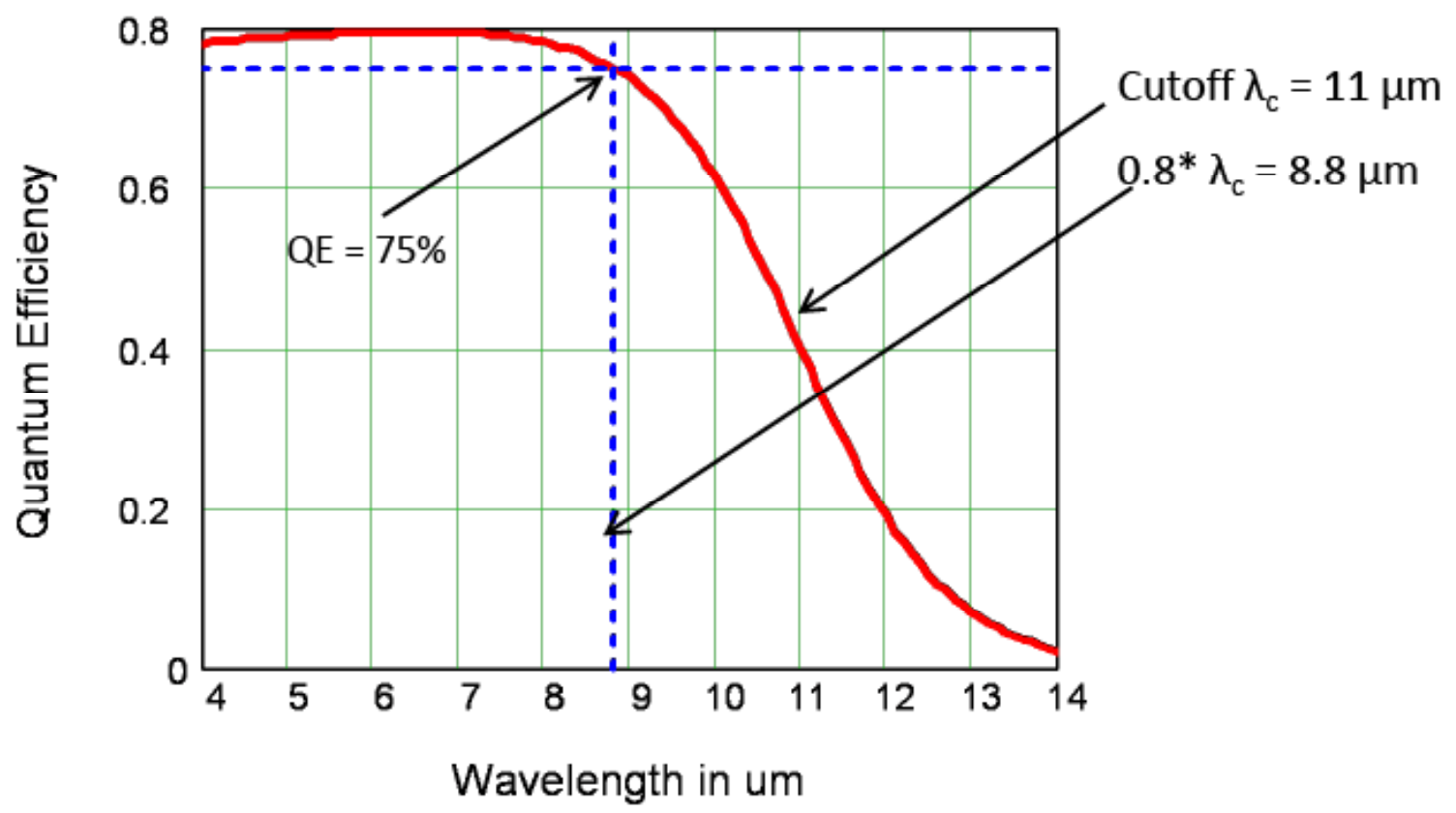

Figure 2: Recommended definition for single-valued quantum efficiency reporting. It is defined as the wavelength that equals to $80 \%$ of the cutoff wavelength. At the cutoff wavelength, the quantum efficiency falls to $50 \%$ from the plateau.

\section{TECHNICAL CHALLENGES AND POTENTIAL SOLUTIONS}

It is important to create more advanced detector architecture designs for single- and dual-band detectors. It is equally important to be able to experimentally verify how well the MBE grown device and its performance follow the designed value. Imperfect control of growth conditions causes actual detector structure deviations from the design in doping profile, junction position, layer thickness, and interface atomic composition. Feedback from experimental data will help device designers to modify the designs and possibly compensate for the imperfect growth conditions. This feedback will also help researchers gain insight into the robustness of the design (i.e., the degree of performance degradation due to small deviations of actual device structure from the design). For dual-band detector design, the current program intention is to adopt bias-switchable, back-to-back diodes designed with single-bump connection to ROICs. This is aimed at minimizing the deleterious surface effect associated with multiple bumps. Detailed discussions about multiple design options with different layer sequences using non-superlattice materials, as well as the etch depth options and spectral cross talk, can be found in the literature. ${ }^{34-36}$ Initial experimental results with antimonide superlattice materials were also reported in the literature. ${ }^{37-38}$ An existing challenge for superlattice dual-band detector design is to accurately control the multiple layer growth and realize the designed band alignment at either voltage bias polarity to achieve high performance at each band, while minimizing electrical and optical cross talk.

Planar detector structure could be an alternative to the current mesa structure to minimize the surface leakage current. Several groups have implemented such structure through ion implantation, diffusion, or ion milling on $\mathrm{HgCdTe}$ and InAlGaAs/InAlAs superlattice materials. ${ }^{39-43} \mathrm{~A}$ recent study considered $\mathrm{Zn}$ diffusion behavior in GaSb for the purpose of fabricating planar $\mathrm{P}$ on $\mathrm{N}$ antimonide superlattice detectors. ${ }^{44}$ Researchers have also studied ways to minimize spatial cross talk and blooming effects, such as using guard rings created by a small doped region between pixels for $\mathrm{HgCdTe}$ 
detectors. ${ }^{45}$ Innovative research to devise ways to implement advanced planar structure on antimonide superlattice is encouraged under the technology program and FastFPA program.

Some of the superlattice detector designs use materials of different bandgaps to form a heterojunction to reduce dark current. Due to imperfect control of doping profile, the detector band alignment may deviate from the design, and as a result a potential barrier or Bratt barrier is formed. ${ }^{46}$ This barrier inhibits minority carrier photocurrent and degrades quantum efficiency. A high reverse bias voltage is usually required to overcome the barrier. This is undesirable because most existing readout chips have a nominal maximum reverse bias around $500 \mathrm{mV}$. More important, the voltagedependent quantum efficiency may cause responsivity nonlinearity as discussed by Krueger. ${ }^{47}$

$$
I=\frac{I_{d}\left(V_{0}\right)-I_{p h}\left(V_{0}\right)}{1+\frac{R_{S}}{R_{d}}-\left.I_{p h}\left(V_{0}\right) R_{s} \frac{\partial \eta}{\partial V}\right|_{V_{0}} / \eta\left(V_{0}\right)}
$$

where

$I$ is the total current;

$I_{d}\left(V_{0}\right)$ is the dark current;

$I_{p h}\left(V_{0}\right) \sim \eta\left(V_{0}\right) \times Q$ is the photocurrent, which is linearly proportional to incident photon flux $Q$ and quantum efficiency $\eta$;

$R_{S}$ is the series resistance; and

$R_{d}$ is the dynamic resistance.

This equation shows that voltage-dependent quantum efficiency gives rise to nonlinearity between the measured current and incident photon flux if $R_{s}$ is finite.

Unipolar barriers, which are designed to block one carrier type (electron or hole) but allow the unimpeded flow of the other, are used in several superlattice detector structures. ${ }^{27,} 17$ They have been shown to be very effective in suppressing dark current. The antimonide superlattice is an ideal material to implement unipolar barrier structure owing to its great flexibility in engineering electronic band structure. Around the intended unipolar barrier area, grading in composition or doping may be used to minimize the unintended barrier structures or misalignment so that they do not impede the flow of photocurrent. Complete knowledge and precise control of doping profile is critical in achieving high-performance unipolar barrier devices reliably and repeatedly.

Applied material research is necessary to further reduce detector dark current density. A large space of growth conditions has been explored to map out the optimum conditions for producing high-performance materials. ${ }^{48-51}$ For example, the control of V/III flux ratio (i.e., As/In and Sb/(Ga+In)) is critical to reducing surface roughness as measured by AFM and to increasing the photoluminescence intensity. The flux ratio also has significant impact on the superlattice lattice constant during MBE growth of superlattice layers. A 10\% deviation of As/In flux ratio from its optimum value could introduce more than $100 \mathrm{ppm}$ of strain. The growth temperature is another critical parameter that has strong influence on defect generation and surface roughness. The optimization of shutter sequences is crucial in controlling interface type and quality. The control of carrier density directly affects the photoluminescence intensity. Advanced insitu monitoring and control of MBE growth conditions have been implemented, and there is room for further improvement. It is recognized that the growth condition is machine dependent. The optimum growth parameters for one MBE machine may be different for another due to its unique setup and offset in calibration. In addition, small researchtype MBE machines may have different sets of issues than large multiwafer production-type MBE machines. For example, effusion cell spitting defects might be more prominent in small machines, while avoiding nonuniformity across all platens positioned at center, inner ring, and outer ring is a unique challenge for multiwafer large machines.

Using advanced semiconductor characterization tools to quantify various aspects of material properties is critical to further improving material quality. Some of these tools are routinely used today: MBE in-situ monitoring, high resolution X-ray diffraction (HRXRD) for measuring peak positions and FWHM; microscopy such as AFM; and detector electrical characterization such as current-voltage, capacitance-voltage, and Hall measurements. These routine characterizations give immediate feedback about material quality and may be used to predict device performance. There is a report that the HRXRD-derived tensile strain versus the GaSb substrate should be limited to $-3000 \mathrm{ppm}$ and the 
compressive strain should be limited to $4000 \mathrm{ppm}$ because beyond these thresholds, growth defects and dislocations due to surface relaxation will appear, and they will increase the surface roughness and destroy the performance of the material. ${ }^{52}$ There is also a report that if the AFM roughness exceeds certain threshold the responsivity decreases sharply. ${ }^{49}$ Further research into the correlation between measured parameters and underlying superlattice structure and material defect needs to be carried out. For example, although it is understandable that small FWHM from X-ray diffraction data should be an indication that good superlattice structure was grown in general, there is no quantitative knowledge of the intrinsic width of superlattice diffraction profile, and it is not clear if and how all contributory components could be de-convolved to establish correlations between X-ray data and material and structure imperfections. It should be acknowledged that because the phase information is lost in the X-ray rocking curve, it is not possible to directly calculate the structure from X-ray data. Instead, dynamical X-ray simulation has to be performed. ${ }^{53}$ For HgCdTe and CdZnTe, studies have shown that in layers having no inclusions or voids, the threading dislocation density is proportional to the square of X-ray FWHM. In addition, misfit dislocation density can be extracted from the variation of FWHM with epitaxial layer thickness. ${ }^{54}$ For superlattice, there is some indication that dislocation density is low or does not play an important role. ${ }^{51}$ Instead, point defects may be the dominant defect. Under our programs, we plan to find ways to characterize various point defects and establish a new set of quantitative correlations.

Some semiconductor characterization tools are not used as often as they should be because the equipment is expensive and thus not widely available or because their use involves more time-consuming efforts. They include scanning electron microscopy (SEM), transmission electron microscopy (TEM), cross-sectional scanning tunneling microscopy (XSTM), cross-sectional transmission electron microscopy (XTEM), photoluminescence (PL), time-resolved and positionresolved PL, electron beam induced current (EBIC), and deep level transient spectroscopy (DLTS). Under the Technology Program and the FastFPA program there is an effort to make some of these advanced material characterization tools more available to wafer growers and FPA fabricators, so that experimental data from different characterization tools, where each tool reveals a particular aspect of material properties, can be systematically correlated. This should help to gain a complete understanding of material properties and devise a way for their improvement. It is recognized that the precision and resolution requirement placed on these modern semiconductor characterization tools are at the cutting edge of today's equipment technology. The community needs to continue to search for new tools and new ways to perform pioneering work in superlattice characterization and device performance correlation. Some of the tools invented for other material systems may not be applicable to superlattice. For example, where etch pit density is used to reveal dislocation density for $\mathrm{HgCdTe}$, there is no crystallographic etching available for superlattice. Innovative techniques are still to be developed for characterizing various superlattice defects.

Comprehensive studies will be carried out to establish correlations between detector performance and underlying measurable material properties. For example, there is a need to investigate the correlation between defect density in superlattice and substrate layers and detector dark current and FPA operability. Although it is logical to associate high defect density with poor detector performance, there is no well-established, one-to-one cause-effect relation of defect types and quantities with deleterious detector performance. Research in $\mathrm{HgCdTe}$ and preliminary superlattice study shows that not all defects cause degradation in electrical performance of the detector. More work is being carried out to study various defects and their contribution to device performance. These correlative studies could also help to establish predictive figures of merit for FPA fabrications.

Systematic investigations will be carried out on minority carrier lifetime and various lifetime-killing mechanisms. Experimental data will be gathered and analyzed on superlattice materials, as well as on component layers such as GaSb and InAs. The relationship between the lifetimes of a superlattice and its component layers is not clear at the moment: that is, it is not clear how interfaces change the superlattice lifetime characteristics. The interface roughness scattering may play an important role on in-plane and vertical direction carrier transport. Substitutional impurities such as Sb-forAs, In-for-Ga, and compositional grading at interfaces caused by $\mathrm{Sb}$ segregation may also have a profound influence that is still to be unveiled. Better understanding of these topics should help to improve quantum efficiency and reduce dark current.

Currently focal plane array fabrication involves several critical processing steps: dry and wet etching of mesa structure, surface passivation, hybridization to ROIC, underfill of epoxy, and substrate thinning or removal. All these processes demand continuous innovation or improvement. A perfect mesa etching process, characterized with sharp and narrow trenches that are uniform, smooth, clean, and without residue, is still to be established. Breakthrough innovations are required in superlattice material passivation; these have a precondition of complete understanding of candidate passivants' thermal, chemical, electrical, and mechanical properties and their interaction with superlattice materials, as 
well as compatible deposition techniques. Substrate thinning or removal and hybridization are still challenging tasks, probably due to the sensitive nature of superlattice material to strains introduced during the process. Proper underfill materials and deposition techniques are required to avoid dark-current increase or quantum-efficiency degradation.

\section{PROGRAM STRATEGY}

A 4-year program, Fabrication of Superlattice Infrared FPA Program (FastFPA), began April 2009. The program goal is to accelerate superlattice technology transition from research institutions to industry and establish domestic industry capability for superlattice infrared focal plane array fabrication. An important feature of this program is a horizontal integration approach for efficient technology development and demonstration. Multiple domestic suppliers are participants, including commercially profitable III-V semiconductor foundries, incumbent and new FPA chip-assembly vendors, and a commercial readout integrated circuit vendor. This arrangement leverages the existing III-V semiconductor industry market exemplified by up-to-date facilities, high-quality and reliable wafer-production lines, and fast turn-around time. It also eliminates the burden of maintaining vertically integrated wafer growth capability within each FPA house. As a result, it is hoped that novel ideas in device structure design and FPA processing will proliferate via industry participation. In the long term, we also hope to reap the benefit of lower cost FPAs and a more sustainable industry base.

As depicted by Figure 3, the program funds three industry groups. The commercial wafer foundries, including Intelligent Epitaxy and IQE Inc., focus on growing high-quality superlattice materials. They initially get proven structure designs and technical help from the trusted entities (i.e., Government-sponsored research institutions NRL and JPL). Under the guidance of trusted entities, they can quickly graduate from growing materials with simple designs and produce materials of more advanced designs. The trusted entities perform a vital technology-transition role by providing designs, transferring MBE growth technology knowhow, fabricating test diodes, fully characterizing wafers and diodes, and offering suggestions for further improvement. The technical goal for this group is to grow high-quality superlattice materials with $8-10$ micron cutoff wavelength at cryogenic operating temperature, with X-ray diffraction FWHM of 20 arcsec, AFM surface roughness of 2 angstrom, and mismatch to substrate $300-1000 \mathrm{ppm}$. Detectors with quantum efficiency of $60 \%$ and dark current density of 1 microampere per square centimeter will be fabricated. Even though the program has only been implemented for a year, tremendous progress has been made. Details can be found in the papers presented in this conference. ${ }^{55-56}$ After 2 years of government funding, the foundries are expected to start providing commercial wafers according to the design given by the FPA vendors.

The FPA fabrication group, which includes BAE Systems, HRL Laboratory, Raytheon Vision Systems, and Teledyne Imaging Scientific, is funded to process superlattice material from wafer foundries and hybridize detector arrays to readout integrated circuit (ROIC). The program goal is to demonstrate three sets of FPAs in 4 years. They include a small-format FPA, a medium-format FPA, and a large-format FPA. As listed in Table 1, the small- and medium-format FPA can be either single band at long-wavelength infrared (LWIR) or dual band at LWIR and mid-wave infrared (MWIR). The large-format FPA will be single band at LWIR. Again, the trusted entities play a pivotal role here by supplying high-quality superlattice wafers during the first 2 years before the foundries develop full capability, characterizing detectors, and transferring detector and FPA processing technology knowhow to the FPA vendors. After being funded for under a year, all vendors have demonstrated ability to produce SLS FPAs, including one that had never made an FPA before. Details can be found in the papers presented in this conference. ${ }^{33,57-58}$

The ROIC development by FLIR Systems is also funded under the FastFPA program. Three sets of ROICs will be produced and provided to the FPA fabrication group in the program. Other FPA vendors can purchase these ROICs as commercial products. Before the FastFPA program, there were no commercially available ROICs suitable for superlattice FPA development-most of the single-color ROICs on the market are for detectors with P-on-N polarity or require a very high R0A product for high injection efficiency. If used with single-band FPA, all three new ROICs can accommodate N-on-P polarity to match with detectors with P-type absorber and minority electron carriers. They can also accommodate P-on-N polarity. The dual-color ROIC is designed to support bias-voltage-selectable back-to-back diode operation and one indium bump per pixel FPA hybridization. 


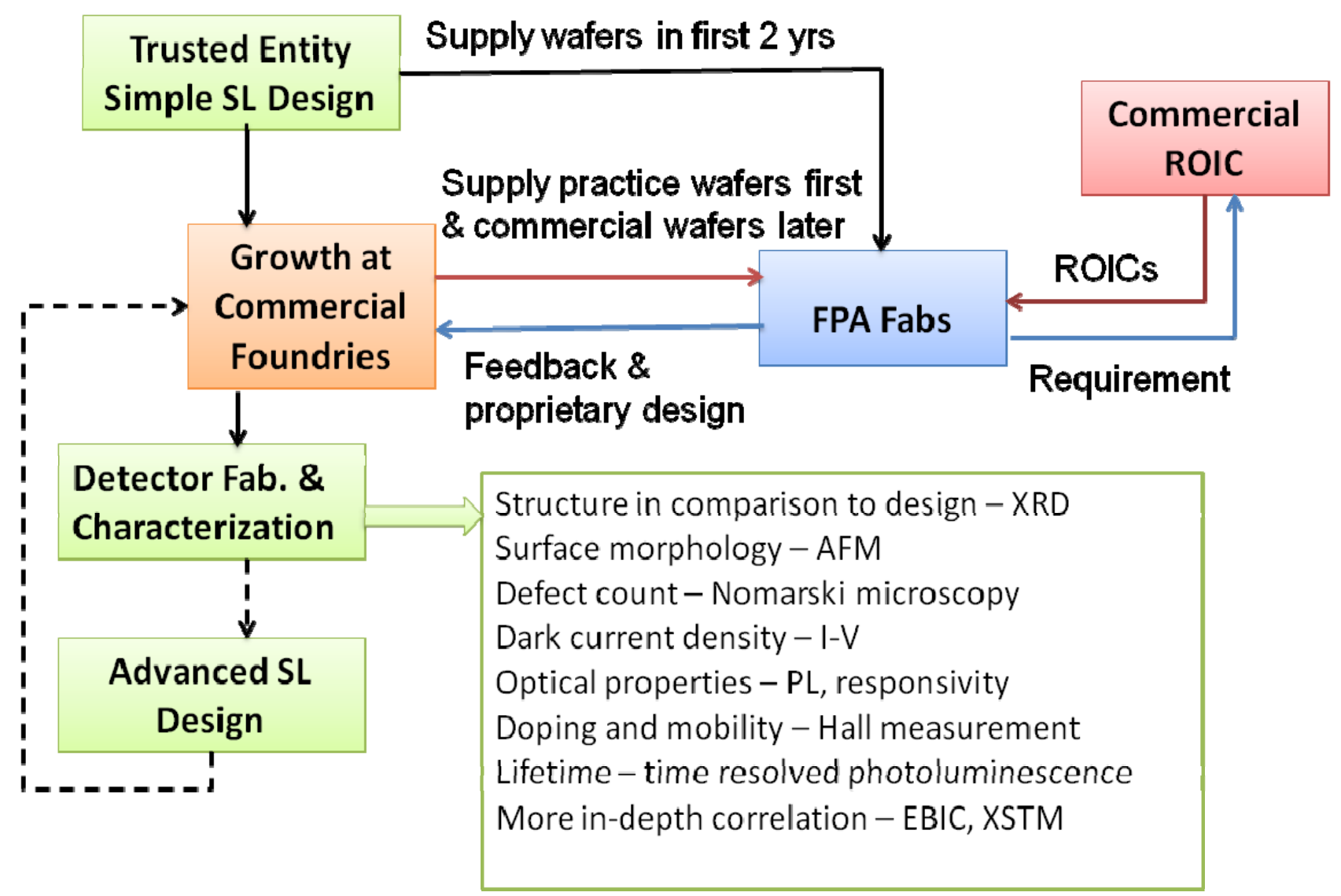

Figure 3: FastFPA program execution model.

Table 1: Three FPAs to be demonstrated under the FastFPA program.

\begin{tabular}{|c|c|l|}
\hline FPA & Format and Pixel Size & \multicolumn{1}{|c|}{ ROIC Polarity } \\
\hline $\begin{array}{c}\text { Small format } \\
\text { Single or dual band }\end{array}$ & $\begin{array}{c}320 \times 256 \\
30 \mu \mathrm{m} \times 30 \mu \mathrm{m}\end{array}$ & $\begin{array}{l}\text { Bias voltage selectable for } \\
\text { either P-on-N or N-on-P }\end{array}$ \\
\hline $\begin{array}{c}\text { Medium format } \\
\text { Single or dual band }\end{array}$ & $\begin{array}{c}640 \times 512 \\
30 \mu \mathrm{m} \times 30 \mu \mathrm{m}\end{array}$ & $\begin{array}{l}\text { Bias voltage selectable for } \\
\text { either P-on-N or N-on-P }\end{array}$ \\
\hline $\begin{array}{c}\text { Large format } \\
\text { Single band }\end{array}$ & $1024 \times 1024$ & N-on-P \\
\hline
\end{tabular}

5. SUMMARY

Much progress has been made in developing antimonide superlattice infrared detectors, as witnessed in the orders-ofmagnitude reduction in dark-current density and substantial improvement in quantum efficiency. Good performance long-wavelength infrared focal plane arrays were also demonstrated, although the dark-current density is about an order of magnitude higher than that of single-element detectors. The community is facing many challenges to further improve the detector and FPA performance. It is necessary to carry out applied research in material growth, processing, and characterization. There is a need to perform correlative studies to establish cause-effect relations among growth conditions, measurable and quantifiable material parameters, processing protocols, detector electrical and optical characteristics, and FPA performance parameters. The community should take advantage of the latest development in semiconductor characterization tools and make them applicable for superlattice device study. Continuous innovation is required in detector array etching, passivation, hybridization, and FPA packaging. The technology program and the new 
FastFPA program will coordinate efforts of research institutions, III-V material foundries, commercial ROIC providers, and FPA fabrication industries. Further reduction in detector and FPA dark current density and improvement in quantum efficiency, as well as meeting other technical challenges, are expected in the next few years. This will ensure the successful demonstration of three sets of FPAs to be accomplished under the FastFPA program.

\section{REFERENCES}

[1] M. Tidrow, L. Zheng, H. Barcikowski, J. Wells, L. Aicheson, Proc. of SPIE Vol. 7298, 72981O-1 (2009); L. Zheng, M. Tidrow, A. Novello, H. Weichel, S. Vohrad, Type II strained layer superlattice: A potential infrared sensor material for space, SPIE Proceedings, Vol. 6900, 69000F-1 (2008).

[2] E. Aifer, J. Tischler, J. Warner, I. Vurgaftman, J. Km, J. Meyer, B. Bennet, L. Whitman, W-structured type-II superlattice based long and very-long wavelength infrared photodiodes, Proceedings of SPIE Vol. 5732, 259 (2005).

[3] P. Delaunay, M. Razeghi, Noise analysis in type-II InAs/GaSb focal plane arrays, J. Appl. Phys. 105, 063110 (2009).

[4] B. Nguyen, D. Hoffman, Y. Wei, P. Delaunay, A. Hood, M. Razeghi, Very high quantum efficiency in type-II InAs/GaSb superlattice photodiode with cutoff of $12 \mu \mathrm{m}$, Appl. Phys. Lett. 90, 231108 (2007).

[5] W. Tennant, D. Lee, M. Zandian, E. Piquette, M. Carmody, MBE HgCdTe Technology: A Very General Solution to IR Detection, Described by ' Rule 07', a Very Convenient Heuristic, J. of Electronic Materials 37, 1406 (2008); W. Tennant, "Rule 07" revisited...still a good heuristic predictor of HgCdTe performance? To be published in $J$. Electronic Materials.

[6] Hooman Mohseni, Yajun Wei, and Manijeh Razeghi, High performance type-II InAs/GaSb superlattice photodiodes, Proceedings of SPIE Vol. 4288, p191 (2001).

[7] Y. Wei, A. Gin, and M. Razeghi, G. Brown, Advanced InAs/GaSb superlattice photovoltaic detectors for very long wavelength infrared applications, Appl. Phys. Lett. 80, p3262 (2002).

[8] Yajun Wei, Aaron Gin, and Manijeh Razeghi, Gail Brown, Type II InAs/GaSb superlattice photovoltaic detectors with cutoff wavelength approaching $32 \mu \mathrm{m}$, Appl. Phys. Lett. 81, p3675 (2002).

[9] Y. Wei, A. Hood, H. Yau, V. Yazdanpanah, and M. Razeghi, M. Tidrow, and V. Nathan, High-performance type-II InAs/GaSb superlattice photodiodes with cutoff wavelength around $7 \mu \mathrm{m}$, Appl. Phys. Lett. 86, 091109 (2005).

[10] Yajun Wei, Andrew Hood, Aaron Gin, Vahid Yazdanpanah, Manijeh Razeghi, and Meimei Tidrow, High performance LWIR Type II InAs/GaSb superlattice photodetectors and infrared focal plane arrays, Proceedings of SPIE Vol 5732, p309 (2005).

[11] Andrew Hood, Manijeh Razeghi, Edward Aifer, and Gail Brown, On the performance and surface passivation of type II InAs/GaSb superlattice photodiodes for the very-long-wavelength infrared, Appl. Phys. Lett. 87, 151113 (2005).

[12] Andrew Hood, Darin Hoffman, Binh-Minh Nguyen, Pierre-Yves Delaunay, Erick Michel, and Manijeh Razeghi, High differential resistance type-II InAs/GaSb superlattice photodiodes for the long-wavelength infrared, Appl. Phys. Lett. 89, 093506 (2006).

[13] Andrew Hood, Pierre-Yves Delaunay, Darin Hoffman, Binh-Minh Nguyen, Yajun Wei, Manijeh Razeghi, and Vaidya Nathan, Near bulk-limited $R 0 A$ of long-wavelength infrared type-II InAs/GaSb superlattice photodiodes with polyimide surface passivation Appl. Phys. Lett. 90, 233513 (2007).

[14] Binh-Minh Nguyen, Darin Hoffman, Pierre-Yves Delaunay, and Manijeh Razeghi, Dark current suppression in type II InAs/GaSb superlattice long wavelength infrared photodiodes with M-structure barrier, Appl. Phys. Lett. 91, 163511 (2007).

[15]Binh-Minh Nguyen, Darin Hoffman, Pierre-Yves Delaunay, and Manijeh Razeghi, Vaidya Nathan, Polarity inversion of type II InAs/GaSb superlattice photodiodes, Appl. Phys. Lett. 91, 103503 (2007).

[16] Binh-Minh Nguyen, Darin Hoffman, Yajun Wei, Pierre-Yves Delaunay, Andrew Hood, and Manijeh Razeghi, Very high quantum efficiency in type-II InAs/GaSb superlattice photodiode with cutoff of $12 \mu \mathrm{m}$, Appl. Phys. Lett. 90 , 231108 (2007).

[17]B.-M. Nguyen, S. Bogdanov, S. Abdollahi Pour, and M. Razeghi, Minority electron unipolar photodetectors based on type II InAs/GaSb/AlSb superlattices for very long wavelength infrared detection, Appl. Phys. Lett. 95, 183502 (2009). 
[18] Darin Hoffman, Binh-Minh Nguyen, Edward Kwei-wei Huang, Pierre-Yves Delaunay, Manijeh Razeghi, Meimei Z. Tidrow, and Joe Pellegrino, The effect of doping the $M$-barrier in very long-wave type-II InAs/GaSb heterodiodes, Appl. Phys. Lett. 93, 031107 (2008).

[19]Edward Kwei-wei Huang, Darin Hoffman, Binh-Minh Nguyen, Pierre-Yves Delaunay, and Manijeh Razeghi, Surface leakage reduction in narrow band gap type-II antimonide-based superlattice photodiodes, Appl. Phys. Lett. 94, 053506 (2009)

[20] Pierre-Yves Delaunaya_and Manijeh Razeghi, Noise analysis in type-II InAs/GaSb focal plane arrays, J. Appl. Phys. 105, $063110(2009)$.

[21]E. H. Aifer, private communications (2001).

[22] E. H. Aifer, E. M. Jackson, G. Boishin, L. J. Whitman, I. Vurgaftman, J. R. Meyer, J. C. Culbertson, and B. R. Bennett, Very-long wave ternary antimonide superlattice photodiode with $21 \mathrm{~mm}$ cutoff, Appl. Phys. Lett. 82, p4411 (2003).

[23] E. H. Aifer, J. G. Tischler, J. H. Warner, I. Vurgaftman, J. C. Kim, J. R. Meyer, B. R. Bennett, and L. J. Whitman, E. M. Jackson and J. R. Lorentzen, W- Structured type-II superlattice based long and very-long wavelength infrared photodiodes, Proceedings of SPIE Vol. 5732, p259 (2005).

[24]E. H. Aifer, J. G. Tischler, J. H. Warner, I. Vurgaftman, W.W. Bewley, J. R. Meyer, J. C. Kim, L. J. Whitman, C. L. Canedy, and, E. M. Jackson, W-structured type-II superlattice long-wave infrared photodiodes with high quantum efficiency, Appl. Phys. Lett. 89, 053519 (2006).

[25] I. Vurgaftman,a_ E. H. Aifer, C. L. Canedy, J. G. Tischler, J. R. Meyer, and J. H. Warner, E. M. Jackson, G. Hildebrandt and G. J. Sullivan, Graded band gap for dark-current suppression in long-wave infrared W-structured type-II superlattice photodiodes, Appl. Phys. Lett. 89, 121114 (2006).

[26] C.L. Canedy, E.H. Aifer, I. Vurgaftman, J.G. Tischler, J.R. Meyer, J.H. Warner, and E.M. Jackson, Antimonide Type-II “'W' Photodiodes with Long-Wave Infrared R0A Comparable to HgCdTe, J. Electronic Mat. Vol 36, p852 (2007).

[27] David Z.-Y. Ting, Cory J. Hill, Alexander Soibel, Jean Nguyen, Sam A. Keo, Jason M. Mumolo, Michael C. Lee, Baohua Yang, and Sarath D. Gunapala, Antimonide superlattice barrier infrared detectors, Proceedings of SPIE Vol. 7419, 74190B-1 (2009).

[28] E. H. Aifer, J. H. Warner, C. L. Canedy, I. Vurgaftman, E. M. Jackson, J. G. Tischler, J. R. Meyer, S. P. Powell, K. Olver, and W. E. Tennant, Shallow-etch mesa isolation of graded bandgap "W" structured type-ii superlattice photodiodes, 2008 U.S. Workshop on the Physics and Chemistry of II-VI Materials, Las Vegas (2008), to be published at $J$. Electronic Materials.

[29] Cory J. Hill, Jian V. Li, Jason. M. Mumolo, Sarath. D. Gunapala, Progress in MBE grown type-II superlattice photodiodes, Proc. of SPIE Vol. 6206, 62060P-1 (2006).

[30] David R. Rhiger, Robert E. Kvaas, Sean F. Harris, Richard E. Bornfreund, Yen N. Thai, Cory J. Hill, Jian V. Li, Sarath D. Gunapala, and Jason M. Mumolo, Progress with type-II superlattice IR detector arrays, Proc. of SPIE Vol. 6542, 654202-1 (2007).

[31] M. Field, G.J Sullivan, and A. Ikhlassi, C. Grein, M.E. Flatté, H. Yang, M. Zhong and M. Weimer, Electrical and Optical Performance of InAs/GaSb Superlattice LWIR Detectors, Proc. of SPIE Vol. 6127, 61270V-1 (2006).

[32] Andrew D. Hood, Allan J. Evans, Amal Ikhlassi, Donald L. Lee, William E. Tennant, 2009 U.S. Workshop on the Physics and Chemistry of II-VI Materials, Chicago, Oct (2009). To be published at J. Electronic Materials.

[33] Andrew D. Hood, Allan J. Evans, Amal Ikhlassi, Gerard J. Sullivan, Eric C. Piquette, Donald L. Lee, William E. Tennant, Igor Vurgaftman, Chadwick L. Canedy, Edward H. Aifer, High-performance LWIR type-II strained layer superlattice focal plane arrays, Paper 7660-50, Proceedings of SPIE Defense, Security, and Sensing Conference (2010).

[34] N.T. Gordon, P. Abbott, J. Giess, A. Graham, J.E. Hails, D.J. Hall, L. Hipwood, C.L. Jones, C.D. Maxey, and J. Price, Design and Assessment of Metal-Organic Vapor Phase Epitaxy Grown Dual Waveband Infrared Detectors, Journal of Electronic Materials, Vol. 36, No. 8, p931 (2007).

[35]E. Blazejewski, J. Arias, G. Williams, W. McLevige, M. Zandian, and J. Pasko, Bias-switchable dual-band HgCdTe infrared photodetector, J. Vacuum Science Technology, B 10, p1626 (1992).

[36] R.A. Coussa, A.M. Gallagher, K. Kosai, L.T. Pham, G.K. Pierce, E.P. Smith, G.M. Venzor, T.J. de Lyon, J.E. Jensen, B.Z. Nosho, J.A. Roth, and J.R.Waterman, Spectral Crosstalk by Radiative Recombination in SequentialMode, Dual Mid-Wavelength Infrared Band HgCdTe Detectors, Journal of Electronic Materials, Vol. 33, No. 6, p517 (2004). 
[37]E. H. Aifer, J. G. Tischler, J. H. Warner, I. Vurgaftman, and J. R. Meyer, C. L. Canedy and E. M. Jackson, Dual band LWIR/VLWIR type-II superlattice photodiodes, Proc. of SPIE Vol. 5783, p112 (2005).

[38] Pierre-Yves Delaunay, Binh-Minh Nguyen, Darin Hoffman, Andrew Hood, Edward Kwei-Wei Huang, Manijeh Razeghi, and Meimei Z. Tidrow, High quantum efficiency two color type-II InAs/GaSb $n-i-p-p-i-n$ Photodiodes, Applied Physics Letters 92, 111112 (2008).

[39] J. M. Arias, J. G. Pasko, M. Zandian, S. H. Shin, G. M. Williams, L. 0. Bubulac, R. E. DeWames, and W. E. Tennant, Planar p-on-n HgCdTe heterostructure photovoltaic detectors, Appl. Phys. Lett. 62 (S), p976 (1993).

[40] G. Bahir, V. Garber, and A. Dust, Characterization of a New Planar Process for Implementation of p-on-n HgCdTe Heterostructure Infrared Photodiodes, Journal of Electronic Materials, Vol. 30, No. 6, p704 (2001).

[41] C.A. Musca, J. Antoszewski, J.M. Dell, L. Faraone, and S. Terterian, Planar p-on-n HgCdTe Heterojunction MidWavelength Infrared Photodiodes Formed Using Plasma-Induced Junction Isolation, Journal of Electronic Materials, Vol. 32, No. 7, 2003.

[42] L. Mollard, G. Destefanis, N. Baier,1 J. Rothman, P. Ballet, J.P. Zanatta, M. Tchagaspanian, A.M. Papon, G. Bourgeois, J.P. Barnes, C. Pautet, and P. Fougeres, Planar p-on-n HgCdTe FPAs by Arsenic Ion Implantation, Journal of Electronic Materials, Vol. 38, No. 8, p1805 (2009).

[43] K. Taguchi, K. Makita, I . Watanabe, M. T S U J I, M. Hayashi, T. Nakata, Superlattice avalanche photodiodes for optical communications, Optical and Quantum Electronics 30, p219 (1998).

[44] Honnavalli R Vydyanath, Investigation of the diffusion behavior of $\mathrm{Zn}$ for the fabrication of planar $\mathrm{P}$ on $\mathrm{n} \mathrm{GaSb}$ based type II superlattice detectors, presentation at the 2009 U.S. Workshop on the Physics and Chemistry of II-VI Materials, Chicago, October (2009).

[45] J. Ziegler, M. Bruder, M. Finck, R. Kreuger, P. Menger, Th. Simon, R. Wollrab, Advanced sensor technologies for high performance infrared detectors, Infrared Physics \& Technology 43 239-243, (2002)

[46] P. Bratt, HgCdTe Heterojunctions, J. Vacuum Science Technology A, 1(3), p1687 (1983).

[47]E. E. Krueger, D. Lee, C. Miller, C. L. Terzis, P. O'Dette, B. Denley, J. Rutter, J. L. Williams and M. B. Reine, $\mathrm{HgCdTe}$ photodiodes with cutoff wavelengths of $17 \mathrm{um}$ at $70 \mathrm{~K}$ for use in high resolution interferometers for remote sensing, SPIE Proceedings Vol. 3122, p355 (1997).

[48] Aifer, E. H., Jackson, E. M., Bennett, B.R, Vurgaftman, I., Meyer, J. R., and Jernigan, G. G., Suppression of bulk defects in antimonide superlattice infrared photodiodes, Mat. Res. Sco. Symp. Proc. Vol. 722, K10.1.1 (2002).

[49]F. Fuchs, L. Burkie, W. Pletschen, J. Schmitz, M. Waither, InAs/Ga1.InSb infrared superlattice diodes: Correlation between surface morphology and electrical performance, SPIE Proceedings Vol. 3794 page 41 (1999).

[50] R. Rehm, M. Walther, J. Schmitz, J. Fleißner, F. Fuchs, J. Ziegler, And W. Cabanski, InAs/GaSb superlattice focal plane arrays for high resolution thermal imaging, Opto-Electron. Rev., 14, no. 1, p19 (2006).

[51] Y. Wei, A. Gin, and M. Razeghi, Quantum photovoltaic devices based on antimony compound semiconductors, Mid-infrared Semiconductor Optoelectronics, p. 515, Springer Series in Optical Sciences (2007).

[52] Binh-Minh Nguyen, Darin Hoffman, Pierre-Yves Delaunay, Edward Kwei-Wei Huang, Manijeh Razeghi, and Joe Pellegrino, Band edge tunability of $M$-structure for heterojunction design in $\mathrm{Sb}$ based type II superlattice photodiodes, Applied Physics Letters 93, 163502 (2008).

[53] B.K. Tanner and M.J. Hill, X-ray double crystal diffractometry of multiple and very thin heteroepitaxial layers, $A d v$. X-ray Anal., 29, 337 (1986).

[54] Junhao Chu and Arden Sher, Physics and Properties of Narrow Gap Semiconductors, ISBN: 978-0-387-74743-9, 2008 Springer Science+Business Media, LLC.

[55] D. Lubyshev, J. Fastenaua, X. Gu, A. Liu, J. Prineas, E. Koerperick, J. Olesberg, E. Jackson, J. Nolde, C. Yi, E. Aifer, MBE Growth of Sb-based Type-II Strained Layer Superlattice Structures on Multi-wafer Production Reactor, paper 7660-47, Proceedings of SPIE Defense, Security, and Sensing Conference (2010).

[56] Paul Pinsukanjana, Kevin P. Clark, Jenn-Ming Kuo, Kevin W. Vargason, Yung C. Kao, Status of multi-wafer production MBE growth of Type-II SL at IntelliEPI, Paper 7660-46, Proceedings of SPIE Defense, Security, and Sensing Conference (2010).

[57] David R. Rhiger, Robert E. Kvaas, Sean F. Harris, Borys P. Kolasa, Cory J. Hill, David Z. Ting, Characterization of barrier effects in superlattice LWIR detectors, Paper 7660-51, Proceedings of SPIE Defense, Security, and Sensing Conference (2010).

[58] Rajesh Rajavel, Sevag Terterian, Brett Z. Nosho, Hasan Sharifi, Fabrication and performance of InAs/GaSb-based superlattice LWIR detectors, Paper 7660-52, Proceedings of SPIE Defense, Security, and Sensing Conference (2010). 